\title{
ADVANCED BWR STABILITY ANALYSIS WITH A REDUCED ORDER MODEL AND SYSTEM CODE
}

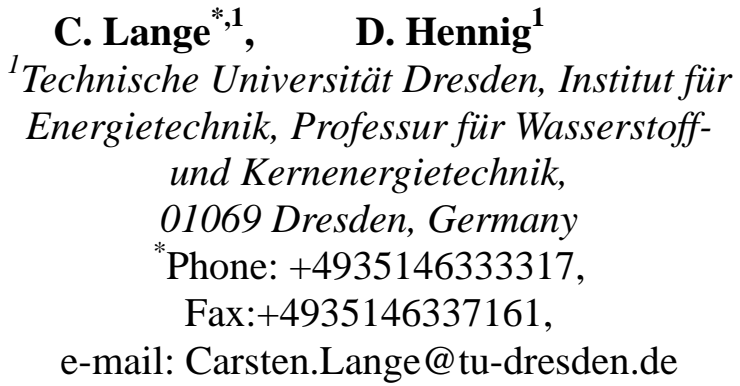

\author{
V. Garcia I Llorens and G. Verdu \\ Departament d'Enginyeria Quìmica i \\ Nuclear, \\ Cami de Vera $s / n$ \\ CP 46022 \\ Valencia \\ Spain \\ e-mail: vigarllo@isirym.upv.es
}

Keywords: BWR, Reduced order model, Drift Flux Model, Homogeneous Equilibrium Model, Stability Analysis, Bifurcation Analysis, Boiling Water Reactors.

\section{ABSTRACT}

The solution manifold of the system of nonlinear differential equations representing a BWR needs to be examined to understand its dynamical behavior. In particular, stable or unstable fixed points and stable or unstable oscillatory solutions (or turning points/saddle node bifurcations) are of paramount interest. In this framework integrated BWR (system) codes and simplified BWR models (reduced order models, ROM) are used together to reveal the solution manifold of the nonlinear differential equations describing the system. This work is a continuation of the previous work at the Paul Scherrer Institute (PSI, Switzerland) and University of Illinois (USA) on this field. The ROM developed at PSI was upgraded by introducing the recirculation loop, subcooled boiling and modification to the feedback reactivity calculation. The upgraded ROM has been coupled with the BIFDD code which performs semi-analytical bifurcation analysis. The
\end{abstract}

methodology and some results of the semi-analytical bifurcation analysis of the modified ROM will be demonstrated and discussed. The stability boundary (SB) and the nature of the Poincarè-Andronov-Hopf bifurcation (PAH-B) are determined and visualized in appropriate two-dimensional parameter maps. Furthermore, for independent confirmation of the results, numerical integrations of the ROM differential equations have been carried out in the MATLAB environment.

\section{INTRODUCTION}

From theoretical and experimental studies, it is well known that for a boiling water reactor (BWR) plant there exists operational points (characterized by a given thermal power, core coolant flow, core inlet subcooling and control rod position) where slowly decaying or non-decaying power oscillations are observed [1-6]. Global or in-phase oscillations and 
regional or out-of-phase oscillations are two kinds of observed power oscillations. In the in-phase mode, the fundamental mode oscillates, while in the out-of-phase mode the first azimuthal mode oscillates: when the power or flow rises in one half of the core, it decreases in the other half in such a way that the total mass flow and the core power remain constant. The physical mechanism behind the stable and unstable oscillatory behavior is based on the coupling between the neutron kinetics and the thermal hydraulics via the void and Doppler feedback reactivity $[5,6]$.

The dynamics of boiling water reactors (BWR) can be described by a system of coupled nonlinear partial differential equations. From the nonlinear dynamics point of view, it is well known that such systems show, under specific conditions, very complex behavior which is reflected in the solution manifold of the corresponding equation system [1-9]. Consequently, to understand the nonlinear stability behavior of a BWR, the solution manifold of the differential equation systems must be examined. In particular, with regard to the existence of operational points where stable and unstable power oscillations are observed, stable or unstable fixed points and stable or unstable oscillatory solutions (or turning points/saddle node bifurcations) are of paramount interest $[2,5,6,10]$.

These investigations have reactor safety relevance because power oscillations could induce undesirable hot spots in a BWR [2]. If the amplitudes become large enough, technical limit values (as critical power ratio) could be exceeded and fuel element failure could be expected. Furthermore, there exist BWR states where unstable limit cycles may occur (in the neighborhood of subcritical bifurcations [7-9]). In this case, small perturbations imposed on the system do not grow (stable). But if a critical perturbation amplitude is exceeded the system behavior becomes unstable. Therefore, conceivably, unstable conditions are not recognized and the operational safety limits could be violated. Hence the methodology of the nonlinear stability analysis of BWR, applied in the current work, should reveal such phenomena.

In the framework of this approach, integrated BWR (system) codes and simplified BWR models are used together to examine the stability characteristic of fixed points and periodic solutions of the nonlinear differential equations describing the stability behavior of a BWR loop [2,3,5,6]. This work is a continuation of the previous work at Paul Scherrer Institute (PSI, Switzerland) and University of Illinois (USA) on this field. The current ROM was extended by adding the recirculation loop and a model which takes into account the effect of subcooled boiling in an approximate manner. Furthermore, a new calculation methodology for the feedback reactivity was implemented. The modified ROM was coupled with the code BIFDD [3, 4-6] which performs semianalytical bifurcation analysis.

This paper presents the motivation and the basic principles of this methodology. In addition to that the recirculation loop model and his influence on the SB and the PAH-B will be demonstrated. The new feedback reactivity calculation methodology and the subcooled boiling model will be presented in separate papers.

\section{METHODOLOGY}

Under variation of one or more selected system parameters (control parameters) a stable fixed point (stationary point) can lose his stability and a Hopf bifurcation occurs (under certain conditions explained later) which results in an isolated stable or unstable periodic solution (also termed stable or unstable limit cycle) of the system equations. The investigation of such nonlinear system behavior is the main objective of nonlinear stability analysis [1-13]. In the following, system codes and reduced order models are briefly characterized.

System codes are computer programs which include detailed physical models of all nuclear power plant components which are significant for a particular transient analysis [2]. Therefore, such detailed BWR models should be able to represent the stability characteristics of a BWR close to the physical reality. Nonlinear BWR stability analysis with the aid of large system codes is currently common practice in many laboratories [2-6]. A particular requirement is the integration of a 3D neutron kinetic model for the core, thereby permitting analysis of regional or higher mode stability behavior [1-3].

A detailed investigation of the complete solution manifold of the nonlinear equations describing BWR stability behaviour by employing system codes needs comprehensive parameter variation studies which require large computational effort. Hence system codes are inappropriate to reveal the complete stability characteristics of a BWR. Therefore, reduced order analytical models become necessary [2-6]. The ROM is characterized by a minimum number of system equations which is mainly realized by reducing the geometrical complexity. One demand on the ROM is that the corresponding equation system should represent the true stability behaviour of a BWR loop. The main advantage of employing ROM is the coupling with methods of semi-analytical bifurcation analysis. In such a methodology the stability properties 
of fixed points and periodic solutions are investigated analytically without the need for solving the system of nonlinear differential equations [2-6].

The main objective of the current work is to combine the system code analysis with the ROM analysis. The intention is, first, to identify the stability properties of certain operational points by performing ROM analysis and, secondly, to apply the system code for a detailed stability investigation in the neighbourhood of these operational points [2]. To this end, plant model data and data characterizing the operational point of a specified BWR plant will be extracted from the system code RAMONA. These data, appropriately recalculated, are ROM inputs. In the first step of the ROM investigation, semi-analytical bifurcation analysis will be performed. As a result, the $\mathrm{SB}$ and the nature of the PAH-B are determined. In the second step, for independent confirmation of the results, numerical integrations of the ROM differential equations will be carried out for specified parameter values $[2-6,10]$.

\subsection{Semi-Analytical Bifurcation Analysis}

Stability analysis is the investigation of the behavior of the dynamical variables after a perturbation is introduced into the dynamical system. If the system is stable, all dynamical variables return to the fixed point (or in a close neighborhood of the fixed point $)^{1}$. If the system is unstable, at least one dynamical variable is diverging in an oscillating or exponential manner. Thereby the SB, which depends on the system parameters, separates the stable fixed points from the unstable fixed points. A comprehensive mathematical description is given in [11-13].

In the framework of the present specific BWR stability research, the semi-analytical bifurcation analysis, the so-called Hopf bifurcations play a dominant role. The occurrence of such type of dynamical bifurcations is ensured by the Hopf theorem [12, 13]. This theorem, which is also called Poincarè-Andronov-Hopf bifurcation theorem, guarantees the existence of stable and unstable periodic solutions of nonlinear differential equations if certain conditions are satisfied [11-13]. For a mathematical description, the autonomous system,

$$
\frac{d}{d t} \vec{X}(t)=\vec{F}(\vec{X}(t), \gamma)
$$

is considered. Thereby, $\vec{X} \in \mathbb{R}^{n}$ is the state vector, $\vec{F}$ ( $\vec{F}: \mathbb{R}^{n} \times \mathbb{R} \rightarrow \mathbb{R}^{n}$ is $C^{\infty}$ ) is a vector field and $\gamma \in \mathbb{R}^{m}$ is a parameter vector (also called control parameter

\footnotetext{
${ }^{1}$ Also called “Ljapunov stability”[12]
}

vector). Let $\vec{X}_{0}$ be the steady state solution $0=\vec{F}\left(\vec{X}_{0}, \gamma\right)$ of Eq.(1) for all $\gamma$ and $J(\gamma)$ be the Jacobian matrix of $\vec{F}$ along the steady state solution [5,10,12]. If the following Hopf conditions are fulfilled:

1) For a critical parameter $\gamma_{c}$ there exists a pair of complex conjugate eigenvalues $\lambda\left(\gamma_{c}\right)= \pm i \omega$,

2) all the other eigenvalues have strictly negative real parts, and

3) $\frac{\partial \lambda\left(\gamma=\gamma_{c}\right)}{\partial \gamma} \neq 0$,

periodic solution of (1) exist at $\vec{X}_{0}$ for $\gamma_{c}$ [5, 10-13].

In order to get information about the stability property of the periodic solution, the (linear) Floquet theory is applied where the so-called Floquet exponent (Floquet parameter) $\beta_{2}$ appears [5] which determines the stability of the periodic solution. If $\beta_{2}<0$, the periodic solution is stable (supercritical bifurcation) while if $\beta_{2}>0$, the periodic solution is unstable (subcritical bifurcation) [5, 10-13].

If the Hopf theorem is satisfied, the nonlinear equation system can be reduced to a two-dimensional nonlinear equation system by applying the center manifold reduction approach [11-13]. The resulting equation system, which represents the dynamical behavior of the complete system of equations in a close neighborhood of the fixed point where the Hopf theorem is fulfilled, will be transformed into the Poincarè normal form [13]. From this equation system parameters (in particular the Floquet exponents) which determine the stability properties of the fixed point, can be extracted numerically.

\subsection{The BIFDD Code}

The code BIFDD was developed to perform bifurcation analysis of ordinary differential equations (ODEs) numerically [5, 8, 10, 13]. For this purpose, a set of nonlinear ODEs and the corresponding Jacobian matrix are code inputs and the critical value of the bifurcation parameter (SB), the nature of PAH bifurcation and the oscillation amplitude will be determined. Thereby, the analytical reduction of the set of nonlinear ODEs on the Poincarè normal form (twodimensional manifold) via the center manifold theorem is carried out numerically $[5,10,13]$.

Hence, a FORTRAN subroutine was written ([26]) to provide the right hand side of the set of nonlinear ODEs as well as the Jacobian matrix ${ }^{2}$ (the

\footnotetext{
2 The right hand side of the set of ODEs and the corresponding Jacobian matrix were derived analytically using the Maple symbolic tool box.
} 
subroutine is called by BIFDD [5]). A main program was written [2-6] that allows selecting any of the design or operational parameters as the bifurcation parameter. The critical value of the bifurcation parameter can be repeatedly calculated by incrementally varying the so-called iteration parameter (second system parameter), leading to a SB in twodimensional space $[2-6,8,10,13]$.

\section{MODEL}

The current BWR reduced order model consists of three coupled sub-models. These are a neutron kinetic model, a fuel heat conduction model and a twochannel thermal-hydraulic model (presented in $[3,5,6])$. In this paper, only the assumptions on which the sub-models are based on are given. In particular, the recirculation loop model is presented.

\subsection{Neutron Kinetics Model}

The neutron kinetics model is based on the following assumptions:

- The neutron kinetics model is based on two energy groups (thermal and fast neutrons).

- Spatial mode expansion approach of the neutron flux in terms of lambda modes ( $\lambda$-modes).

- Only the first two modes (fundamental and the first mode) are considered [7,9,14,15].

- Only an effective one group of delayed neutron precursors is considered.

- The contribution of the delayed neutron precursors to the feedback reactivity is neglected [3].

Taking into account these assumptions, four modal kinetic equations could be developed, coupled with the equations of the heat conduction and the thermal-hydraulic via the feedback reactivity terms (void and Doppler feedback reactivities). Three feedback reactivity calculation methodologies were developed which are presented in a separate paper [18].

\subsection{Fuel Rod Heat Conduction}

The heat conduction model, completely adopted from Karve et al. [17], is based on the following assumptions:

- Two axial regions, corresponding to the single and two-phase regions, are considered,

- three distinct radial regions, the fuel pellet, the gap and the clad are modeled in each of the two axial regions,

- azimuthal symmetry for heat conduction in the radial direction is assumed,
- heat conduction in the z-direction is neglected,

- time-dependent, spatially uniform volumetric heat generation is assumed.

These assumptions result in a one-dimensional (radial) time dependent partial differential equation (PDE). By assuming a two-piecewise quadratic spatial approximation for the fuel rod temperature, the PDE can be reduced to a system of ODEs by applying the variation principle approach. A detailed derivation is presented in [17].

\subsection{Thermal-hydraulic Model}

The thermal hydraulic behavior of the BWR is represented by two heated channels coupled by the neutron kinetics [2-6,9] and by the recirculation loop. This sub-model is based on the following assumptions:

- The heated channel, which has a constant flow cross section, is divided into two axial regions, the single and the two-phase region.

- All thermal hydraulic values are averaged over the flow cross section

- The dynamical behavior of the two-phase region is presented by a drift flux model (DFM) where mechanical non equilibrium (difference between the two phase velocities, and a radial non-uniform void distribution is considered) is assumed (the DFM represents the stability behavior of the twophase more accurately than a homogeneous equilibrium model, in particular for high void content).

- The two phases are assumed to be in thermodynamic equilibrium.

- The system pressure is considered to be constant.

- The fluid in both axial regions and the downcomer is assumed to be incompressible.

- The following terms are neglected in the energy balance: the kinetic energy, potential energy, pressure gradient, friction dissipation.

- The PDEs (three-dimensional mass, momentum and energy balance equation) are converted into the final ODEs by applying the weighted residual method in which spatial approximations (spatially quadratic but time-dependent profiles) for the single phase enthalpy [17] and the two-phase quality are used (is equivalent to a coarse grained axial discretization).

- The downcomer (constant flow cross section) region is considered to be a single phase region. 
- All physical processes which lead to energy increase and energy decrease are neglected in the downcomer.

- $\quad$ The pump head due to the recirculation pumps is considered to be constant $\left(\Delta P_{\text {head }}=\right.$ const $)$

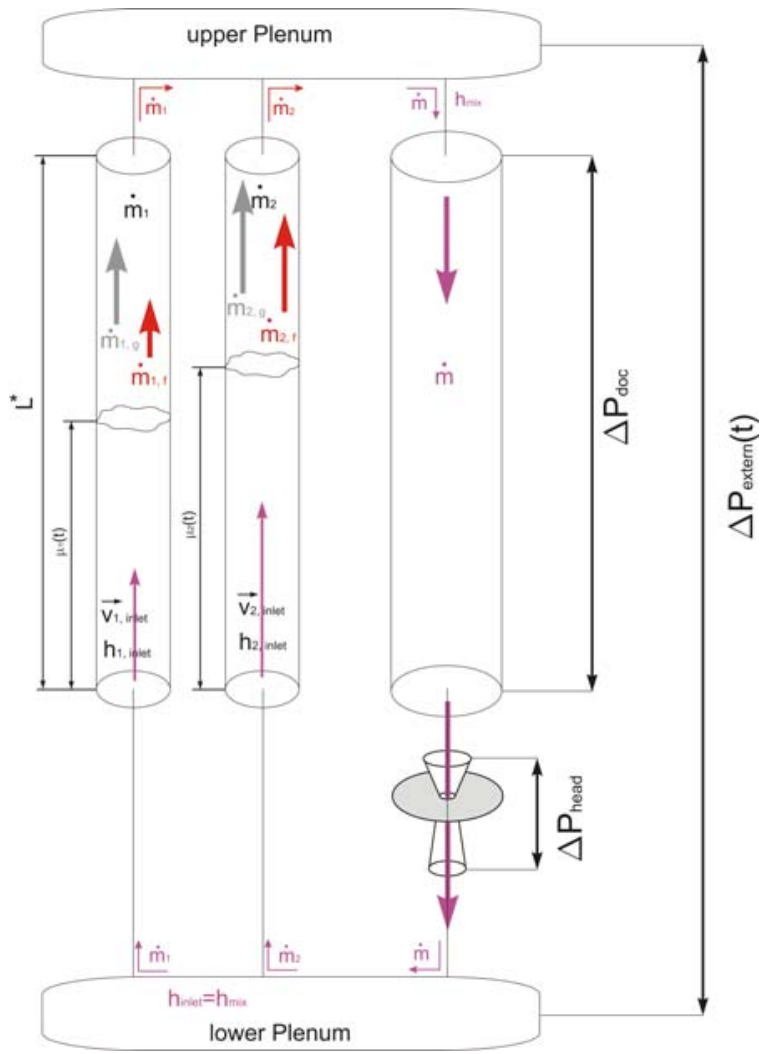

Figure 1: $\quad$ Schematic sketch of the thermal hydraulic two-channel model with outer loop.

Figure 1 depicts a schematic sketch of the thermal hydraulic model including the recirculation loop. The sub-model consists of three parts. These are the two heated channels and the downcomer section. The common lower plenum and the common upper plenum are only shown to indicate that all channels are coupled hydraulically.

The coolant enters the core channel $i$ inlet (single phase region) with the inlet velocities $\mathrm{v}_{i, \text { inlet }}$ and the inlet enthalpy $h_{\text {inlet }}=$ const (is a boundary condition) and the heat is released by nuclear fissions in the fuel, conducted and convected to the coolant. At a certain axial level (boiling boundary $\mu(t)$ ), where the coolant reaches the saturation state, the coolant starts to boil. Above the boiling boundary (two-phase region), the coolant is a mixture of water and steam. Because of the thermodynamic equilibrium between the two phases, the heat generated in the fuel is completely used for steam production.

A comprehensive mathematical description of the single phase and two-phase equations of the current thermal hydraulic heated channel model is given in [3, $5,6]$. In the context of the present paper only the mathematical background of the recirculation loop is given.

The original ROM developed at PSI used a fixed total pressure drop with respect to time $D P_{\text {extern }}=$ const as a boundary condition (equation (3)). According to

$$
\begin{aligned}
& \oint \frac{\partial p}{\partial z} d z=0=\int_{0}^{1} \frac{\partial p_{h_{-} c h}}{\partial z} d z+\int_{1}^{0} \frac{\partial p_{\text {recirc }}}{\partial z} d z \\
& \Rightarrow \quad 0 \approx \int_{0}^{1} \frac{\partial p_{h_{-} c h}}{\partial z} d z-\Delta P_{\text {extern }}
\end{aligned}
$$

the outer loop (second integral in (2)) was replaced by the boundary condition (first integral represents the pressure drop of the heated channel). This is a reasonable approximation to represent the real stability behavior in an out-of phase oscillation mode. But note, in this case the stability behavior of the in-phase oscillation mode can not be simulated correctly. Hence, the ROM was extended by a recirculation loop model.

\section{Recirculation Loop Model}

In the following, a short description of the recirculation loop model is given. In this notation, an asterisk on a variable or parameter indicates the original dimensional quantity while any quantity without an asterisk is dimensionless.

The mass balance of the downcomer can be written as

$$
\frac{\partial}{\partial z} \dot{m}_{t o t}^{*}(t)=0
$$

and the total mass flow $\dot{m}_{\text {tot }}^{*}(t)$

$$
\dot{m}_{\text {tot }}^{*}(t)=\sum_{n} \dot{m}_{n}^{*}(t)=-\rho_{f}^{*}\left[\sum_{n} A_{n, \text { inlet }}^{*} \mathrm{v}_{n, \text { inlet }}^{*}(t)\right]
$$

can accordingly be expressed by the sum of the core channel mass flows $\dot{m}_{n}^{*}(t)$, because the coolant in all hydraulic regions are considered to be incompressible. Thereby, $n$ is the channel number and $A_{n \text {,inlet }}^{*}$ is the flow cross section of the $n$-th heated channel.

The energy balance of the downcomer is reduced to a boundary condition $h_{\text {inlet }}^{*}=h_{\text {doc } \text { inlet }}^{*}=$ const because energy gain and energy loss are neglected.

The momentum balance of the downcomer can be written as 


$$
\begin{aligned}
& -\frac{\partial p_{d o c}^{*}}{\partial z^{*}}=\frac{\partial}{\partial t^{*}}\left(\frac{\dot{m}_{t o t}^{*}(t)}{A_{d o c}^{*}}\right)+\frac{f_{1 \Phi}^{*}}{2 \rho_{f}^{*} D_{d o c}^{*}}\left(\frac{\dot{m}_{t o t}^{*}(t)}{A_{d o c}^{*}}\right)^{2} \\
& +g^{*} \rho_{f}^{*}
\end{aligned}
$$

where the term on the left hand side describes the pressure drop in the downcomer, the first term on the right hand side describes the pressure drop due to inertial effects of the coolant, the second term states the downcomer friction and the last term is the gravity term. Substituting of (5) in (6) and transforming in dimensionless form lead to

$$
\begin{aligned}
& -\frac{\partial p_{d o c}}{\partial z}=-A_{o l}\left(\sum_{n} \frac{\partial}{\partial t} \mathrm{v}_{n, \text { inlet }}(t)\right) \\
& -N_{f 1 \Phi} A_{o l}{ }^{2} D_{o l}\left[\sum_{n} \mathrm{v}_{n, \text { inlet }}(t)\right]^{2}+F r^{-1}
\end{aligned}
$$

where $A_{o l}$ and $D_{o l}$ are defined as $A_{o l}=A_{\text {inlet }}^{*} / A_{d o c}^{*}$ and $D_{o l}=D^{*} / D_{d o c}^{*}$.

The ODEs for the channel inlet velocities $\mathrm{v}_{n \text {,inlet }}(t)$ are determined by expression (2) in which the pressure drop over the recirculation loop is given by

$$
\int_{1}^{0} \frac{\partial p_{\text {recirc }}}{\partial z} d z=\int_{1}^{0} \frac{\partial p_{d o c}}{\partial z} d z-\Delta P_{\text {head }} \text {. }
$$

The evaluation of equation (2) with expression (8) was performed by using the symbolic toolbox of MATLAB. The final ODE for the $n$-th heated channel can be written as

$$
\begin{aligned}
& \frac{d}{d t} \mathrm{v}_{n, \text { inlet }}(t)=A_{n}(t)-B_{n}(t) \cdot\left(\sum_{n} \frac{d}{d t} \mathrm{v}_{n, \text { inlet }}(t)\right) \\
& -B_{n}(t) N_{f 1 \Phi} A_{o l} D_{o l}\left(\sum_{n} \mathrm{v}_{n, \text { inlet }}(t)\right)^{2}
\end{aligned}
$$

where $A_{h}(t)$ is defined as

$$
\begin{aligned}
& A_{n}(t)=\frac{1}{f f_{n, 14}(t)}\left[f f_{n, 11}(t) \frac{d \mu_{n}(t)}{d t}+f f_{n, 12}(t) \frac{d N_{n, p c h}(t)}{d t}\right](10) \\
& +\frac{1}{f f_{n, 14}(t)}\left[f f_{n, 13}(t)+1+F r \cdot \Delta P_{\text {head }}\right]
\end{aligned}
$$

and $B_{n}(t)$ is defined as $B_{n}(t)=F r A_{o l} / f f_{n, 14}(t)$. Thereby, the time dependent intermediate terms $f f_{n, 11}(t), \quad f f_{n, 12}(t), \quad f f_{n, 13}(t)$ and $f f_{n, 14}(t)$ are complicated expressions calculated in [3]. As expected, each ODE for $\mathrm{v}_{\text {ninlet }}(t)$ is hydraulically coupled because of (5) with all the other heated channels. Because of practical relevance, the ODEs for $\mathrm{v}_{n, \text { inlet }}(t)$ was written separately for the one- and the two-heated channel cases. The result for the oneheated- channel case is

$$
\frac{d}{d t} \mathrm{v}_{\text {inlet }}=\frac{1}{1+B}\left[A+B N_{f 1 \Phi} A_{\text {ol }} D_{\text {ol }}\left(\mathrm{v}_{\text {inlet }}\right)^{2}\right]
$$

and the ODEs for $\mathrm{v}_{n \text {,inlet }}(t)$ in the two-heated-channel case $(n=1,2)$ can be written as

$$
\begin{aligned}
& \frac{d}{d t} \mathrm{v}_{1, \text { inlet }}=\frac{-A_{2} \cdot B_{1}+A_{1} \cdot B_{2}+A_{1}}{1+B_{1}+B_{2}} \\
& +\frac{B_{1} N_{f, 1 \Phi} A_{o l} D_{o l}}{1+B_{1}+B_{2}}\left[\mathrm{v}_{1, \text { inlet }}+\mathrm{v}_{2, \text { inlet }}\right]^{2} \\
& \frac{d}{d t} \mathrm{v}_{2, \text { inlet }}=\frac{-A_{1} \cdot B_{2}+A_{2} \cdot B_{1}+A_{2}}{1+B_{1}+B_{2}} \\
& +\frac{B_{2} N_{f, 1 \Phi} A_{o l} D_{o l}}{1+B_{1}+B_{2}}\left[\mathrm{v}_{1, \text { inlet }}+\mathrm{v}_{2, \text { inlet }}\right]^{2} .
\end{aligned}
$$

From the physical point of view, if the downcomer cross section is increased ( $A_{o l}$ decreases), inertial effects of the downcomer mass flow decrease which lead to a constant external pressure drop. Consequently, if the ratio $A_{o l}$ is zero $\left(A_{o l}=0\right)$ inertial effects of the downcomer vanish and (9) is reduced to $\dot{\mathrm{v}}_{n, \text { inlet }}=A_{n}$ (original ODE) which is the result of expression (3) evaluated in [3] (final ODEs for the heated channel inlet velocities presented in $[3,5,6]$ with $D P_{\text {extern }}=F r^{-1}+\Delta P_{\text {head }}$ ).

\subsection{Summary of the Model}

The dynamical system of the reduced BWR model consists of 22 ODEs, four from the neutron kinetics model, eight to describe the fuel rod heat conduction (two equations for each phase, in each channel) and ten that describe the thermal hydraulic model (five for each channel) $[3,5,6]$. Consequently $\vec{X}(t)$ is a vector of 22 phase variables presented in [3, 5, 6]. For the current parameter variation study, the subcooling number $N_{\text {sub }}$ and the phase change number $N_{p c h}$ are of interest. All the other parameters, the operating and design parameters which are the elements of the parameter vector $\gamma$, are considered to be constants $[3,5,6]$ for the stability analysis.

\section{RESULTS AND DISCUSSIONS}

The first investigation with the new ROM was devoted to analyze the influence of the model modification on the SB and the PAH-B characteristics. Because of effects superposed (in particular, global plus regional power oscillation) in the thermal hydraulic two channel case, the first sensitivity study of the impact of the recirculation loop model on the stability properties was first concentrated on a thermalhydraulic one heated channel model (five equation system) in the homogeneous equilibrium (HEM) limit. The use of the HEM limit is justified by the fact that the principal effects of the recirculation loop model on 
the stability property is the same as in the DFM, and differences are only quantitative not qualitative.

In general, the SB can be represented in various operating parameter and/or phase variable planes [3, 5 , $6]$. For the stability and bifurcation analysis with the thermal-hydraulic single heated channel model the stability boundaries were calculated in the $N_{\text {sub }}-N_{p c h}$ operating parameter plane. The subcooling number $N_{\text {sub }}$ represents the core inlet subcooling and appears as a boundary condition in the single phase energy equation. The phase change number (also called Zuber number) scales the phase change due to the heat addition into the coolant of the heated channel. The $N_{\text {sub }}-N_{p c h}$-space shows the thermodynamic state within the heated channel. In face of this the $N_{\text {sub }}-N_{\text {pch }}$-parameter space is often used in the literature as stability map [3-6].

The impact of the subcooled boiling phenomenon and the influence of the new feedback reactivity calculation methodology on the stability properties are presented in separate papers.

4.1. Effects of the Recirculation Loop on the Stability Boundary and Bifurcation Characteristics of a Thermal Hydraulic Single Heated Channel Model

In this section, the impact of the recirculation loop (modified momentum balance) on the SB and nature of the periodic solution of the thermal hydraulic single heated channel model in the HEM limit is performed. To this end, the ratio $A_{\text {ol }}$ (see equation (9)) was varied in small steps (which corresponds to the variation of the downcomer flow cross section $A_{d o c}^{*}$ ) and semianalytical bifurcation analysis was carried out by employing BIFDD. Thereby the bifurcation parameter is $N_{p c h}$ and the iteration parameter is $N_{\text {sub }}$. By setting $D_{o l}=0$, downcomer friction is not considered in (11). Because of practical relevance the ratio $A_{o l}$ and $N_{\text {sub }}$ were varied in the interval $A_{o l} \in[0.0, \ldots, 2.0]$ and $N_{\text {sub }} \in[0.1, \ldots, 4.0]$, respectively.

Figure (2) shows SBs in the $N_{\text {sub }}-N_{p c h}$-parameter space for different $A_{o l}$ values calculated by employing semi-analytical bifurcation analysis. The stability boundaries shift to the right hand site, thus increasing the area of the stability domain, for increasing $A_{o l}$ values. From the stability point of view, the number of stable fixed points increases. According to this, the system becomes more stable.

The bifurcation characteristics for the different $A_{o l}$ values are shown in figure (3). The number of subcritical fixed points (unstable limit cycles) decreases for increasing $A_{o l}$ values. This fact has safety relevance because a subcritical fixed point may deceptively appear as a stable fixed point if the perturbation is sufficiently small. But if the disturbance overcomes the critical amplitude, the fixed point becomes unstable.

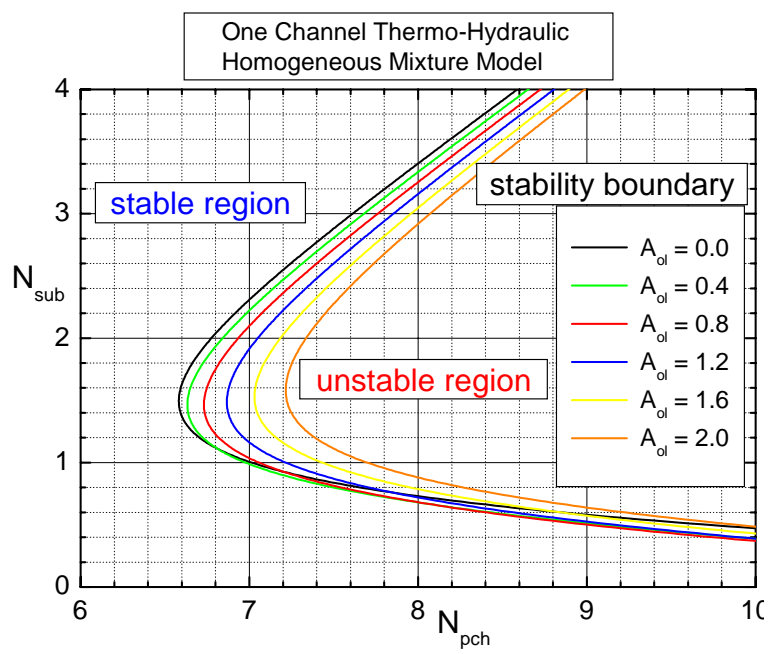

Figure 2: Stability boundaries in the $N_{\text {sub }}$ $N_{p c h}$-parameter space for different ratios $A_{o l} \in[0, \ldots, 2]$.

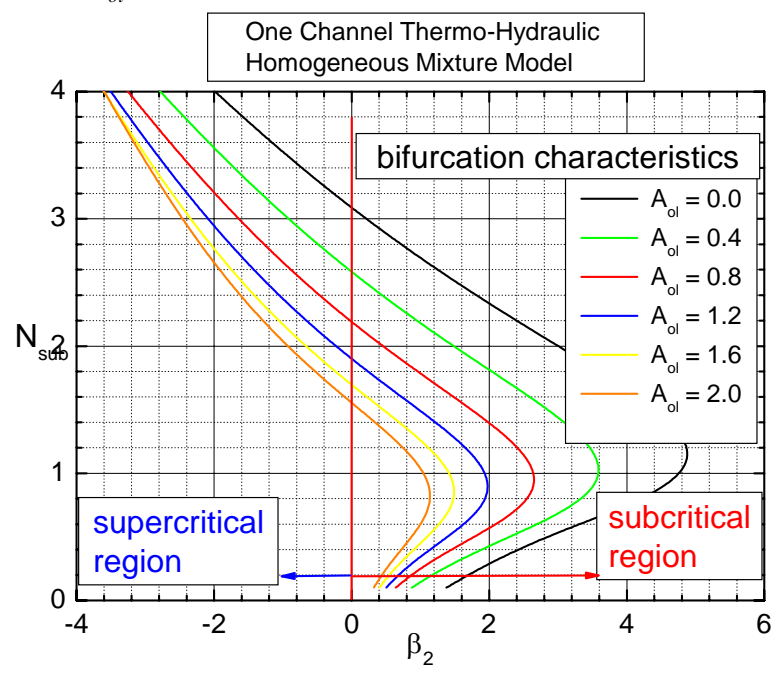

Figure 3: $\quad$ Poincarè-Andronov-Hopf bifurcation characteristic in the $N_{\text {sub }}-\beta_{2}$-parameter ( $\beta_{2}$ Floquet parameter, see chapter 2.1) space for different ratios $A_{o l}$ with $A_{o l} \in[0, \ldots, 2]$

To analyze the influence of the downcomer friction separately the ratio $D_{o l}$ was varied in $D_{o l} \in[0.0, \ldots, 0.14]$ (practical values are placed in the region $\left.D_{o l} \in[0.02, \ldots, 0.04]\right)$ where $A_{o l}$ was set $A_{\text {ol }}=1.2$. 
Figures (4) and (5) present the stability and bifurcation results for the $D_{o l}$ variation. Figure (4) clearly shows that the stability boundaries shift to the left hand side for increasing $D_{o l}$ values. Consequently, the system becomes more unstable. On the other hand, the number of subcritical fixed points decreases for increasing $D_{o l}$ values as shown in figure (5). But both figures also show that the stability behavior of the dynamical system is not sensitive to the $D_{o l}$ variation. Concerning practical values for $D_{o l}$ the downcomer friction can be neglected in further investigations.

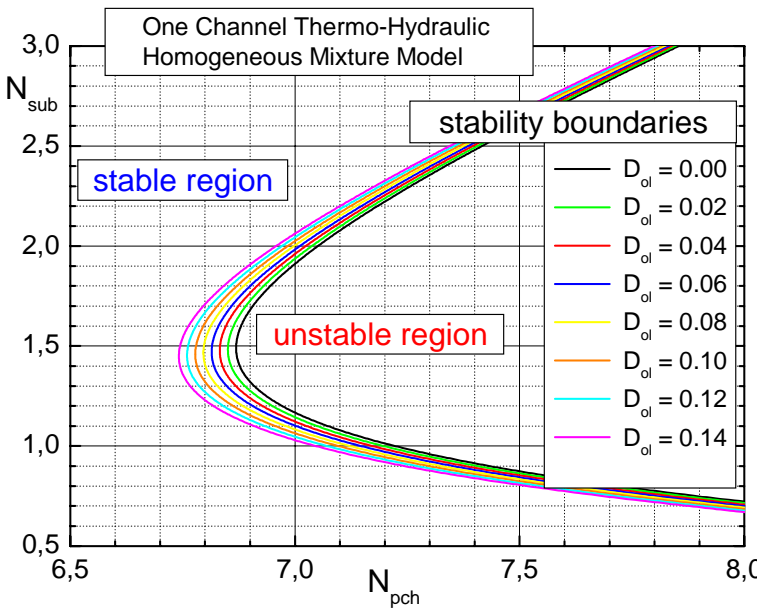

Figure 4: Stability boundaries in the $N_{\text {sub }}$ $N_{p c h}$-parameter space for different ratios $D_{o l}$ with $D_{o l} \in[0.0, \ldots, 0.14]$.

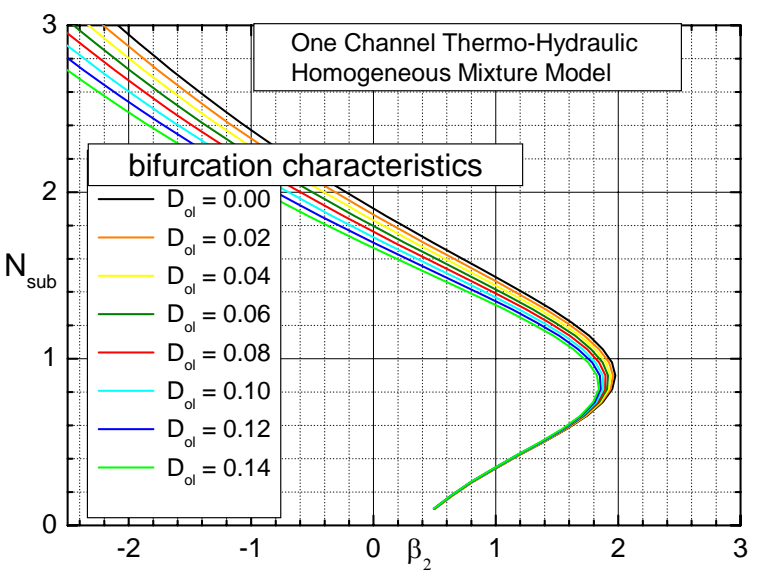

Figure 5: $\quad$ Poincarè-Andronov-Hopf bifurcation characteristics in the $N_{\text {sub }}-\beta_{2}$-parameter space for different ratios $D_{o l}$ with $D_{o l} \in[0.0, \ldots, 0.14]$.
The analyzed results confirm the fact that the recirculation loop model is an essential element in the BWR-ROM. In particular, for the correct presentation of the stability behavior of global power oscillations the external loop should always be considered.

The dominant term in the external loop model (momentum balance) is the inertial term. On the other hand, the downcomer friction has a very small impact on the stability behavior. Consequently, it can be neglected in further stability investigations.

\subsection{Numerical Integration}

Semi-analytical bifurcation analysis is only valid in the vicinity of the SB [3, 5, 6, 12, and 13]. Hence, to get information of the stability behavior beyond the local bifurcation findings numerical integration of the set of the ODEs is necessary. In addition to that the predictions of the semi-analytical bifurcation analysis can be confirmed independently by employing numerical integration. To this end, the ODEs are integrated in the MATLAB environment, where a Runge-Kutta method was used.

The aim of this section is to show the numerical integration method by means of the thermal-hydraulic single heated channel model in a close neighborhood of two points, defined in figure 6 and 7, on the SB.

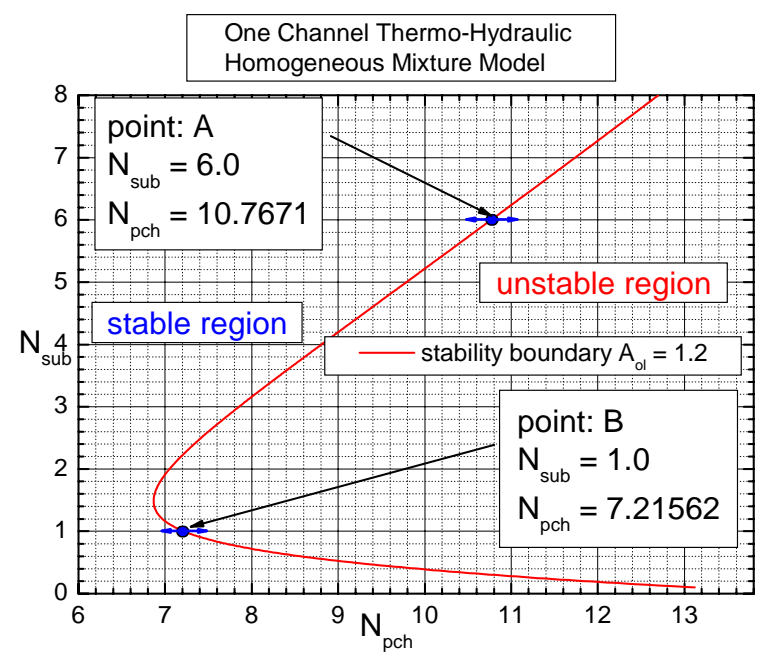

Figure 6:

Definition of two points, $A$ and $B$, on the SB 


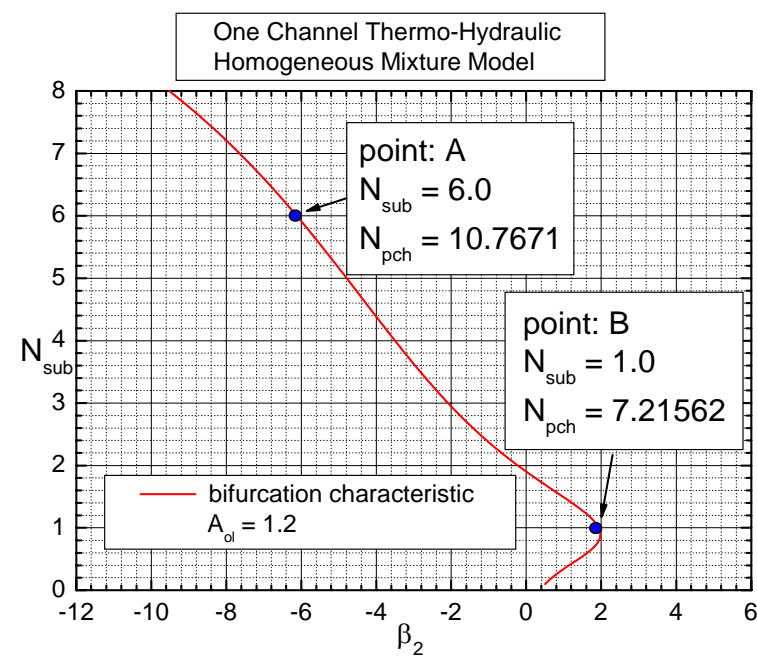

Figure 7: The corresponding points in the bifurcation characteristic map.

Numerical integration results with parameter configurations corresponding to points $A$ and $B$ are presented next.

Analysis in point $A$ : On the left hand side of point $A$, stable fixed points are predicted. For the analysis in this region the phase change number was varied form the critical value $N_{p c h}=10.7671$ to $N_{p c h}=10.7$ and a perturbation in the inlet velocity $\delta \mathrm{v}_{\text {inlet }}$ was introduced to the system (steady state). Figure 8 presents the time evolution of $\mathrm{v}_{\text {inlet }}$. It is clearly shown that the system is stable.

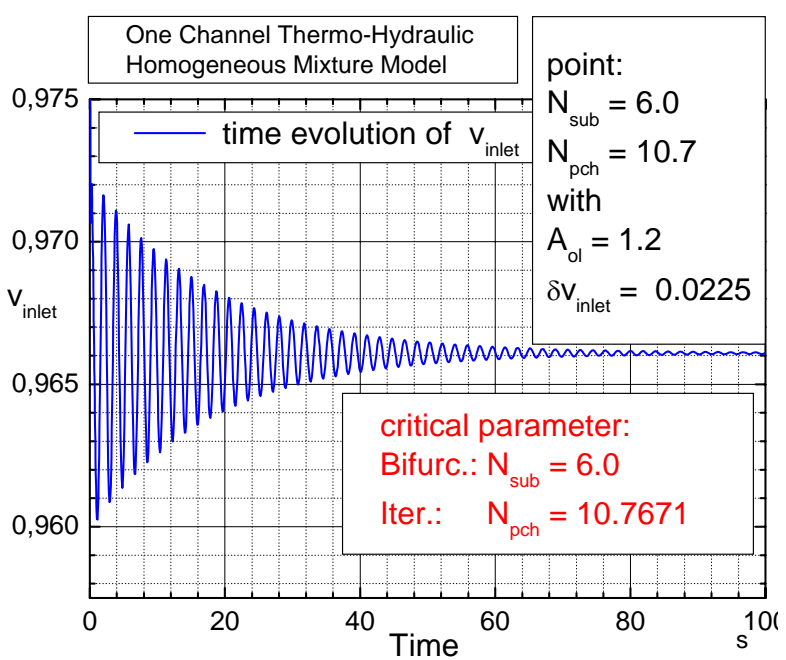

Figures 8: Time evolution of $\mathrm{v}_{\text {inlet }}$. The system is stable on the left hand side of the SB.
On the right hand side of the SB, stable limit cycles of the dynamical system are predicted. For the analysis in this region, $N_{p c h}$ was changed from the critical value $N_{p c h}=10.7671$ to $N_{p c h}=10.78$ and the same perturbation amplitude $\delta \mathrm{v}_{\text {inlet }}$ was introduced into the system. The result is presented in figure 9. As expected, the stable periodic solution of the dynamical system in this point could be confirmed by numerical integration.

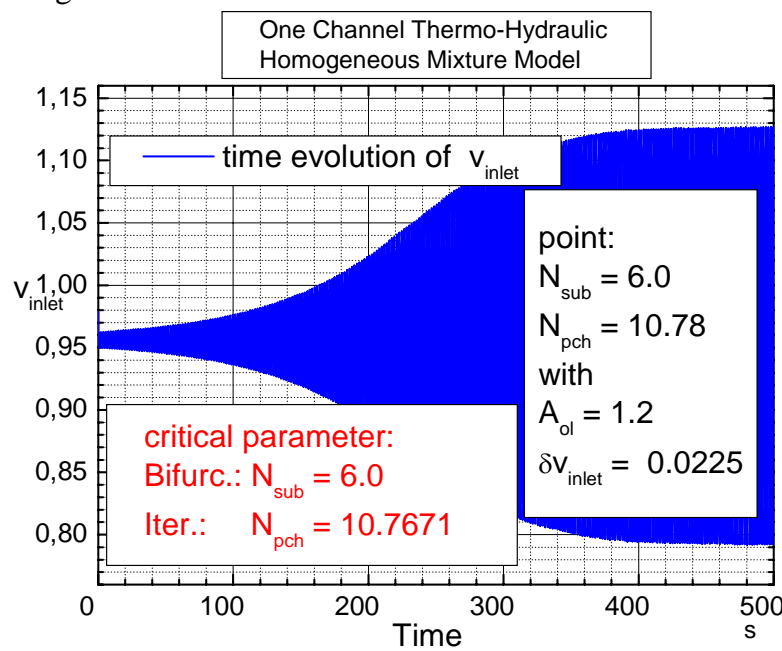

Figure 9: Time evolution of $\mathrm{v}_{\text {inlet }}$. The dynamical system has a stable periodic solution on the right hand side of the SB.

Analysis in point $B$ : A subcritical bifurcation is predicted at point $B$. Consequently, unstable limit cycles are expected on the stable side of the SB. For the numerical integration, $N_{p c h}$ was changed from the critical value $N_{p c h}=7.21562$ to $N_{p c h}=7.2151$. In the first step of the analysis, a perturbation amplitude of $\delta \mathrm{v}_{\text {inlet }}=0.01$ was introduced into the dynamical system and numerical integration was performed. The result is shown in figure 10 . In the second step, a six times larger perturbation amplitude $\delta \mathrm{v}_{\text {inlet }}=0.06$ was introduced into the system which has the same parameter configuration. The result is shown in figure 11. The result, shown in figure 10 and figure 11, confirms the dynamical behavior predicted in the semi-analytical bifurcation analysis. The system is stable for small amplitudes and unstable for large amplitudes.

On the right hand side of point $B$ the system is unstable. In the scope of this paper, results of this region are not presented. 


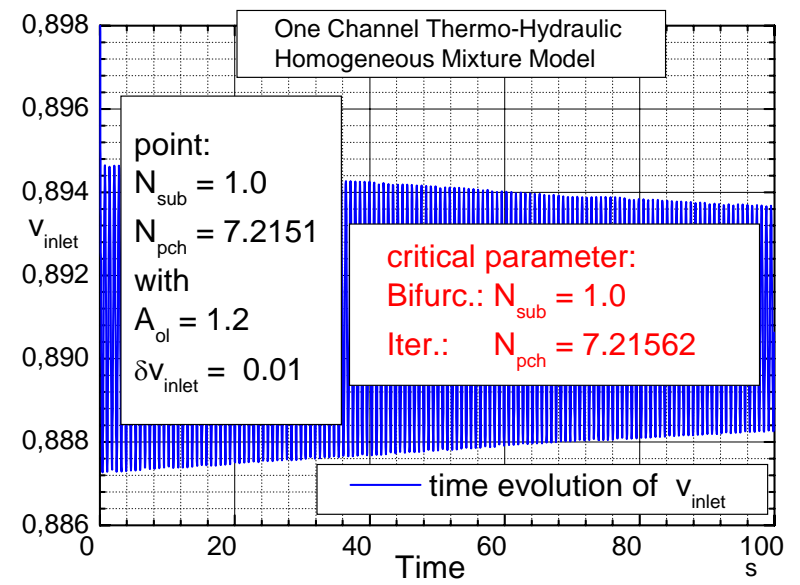

Figure 10: Time evolution of $\mathrm{v}_{\text {inlet }}$. The system is stable for the relative small perturbation amplitude $\delta \mathrm{v}_{\text {inlet }}=0.01$.

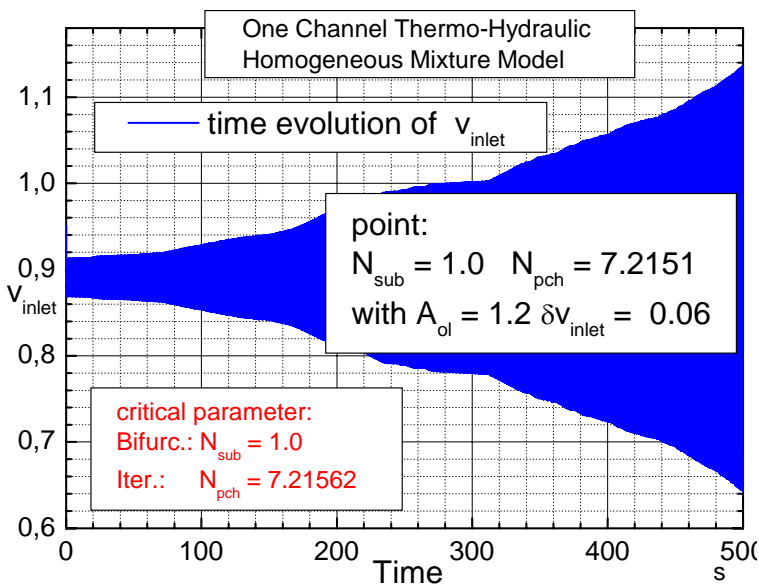

Figure 11: Time evolution of $\mathrm{v}_{\text {inlet }}$. The system is unstable for the relative large perturbation amplitude of $\delta \mathrm{v}_{\text {inlet }}=0.06$.

\section{SUMMARY AND CONCLUSIONS}

In this paper, a recirculation loop model is introduced in the BWR reduced order model. First investigations are devoted in the influence of the model modification on the SB and PAH-B characteristics. To this end, a thermal hydraulic single heated channel model is employed to study the impact of the recirculation loop on the stability properties.

Stability and bifurcation analyses were performed by employing the bifurcation analyses code BIFDD. The SBs and the nature of the PAH-Bs are determined and visualized in a suitable two-dimensional parameter state space.
The study of the impact of the recirculation loop on the SB and PAH-B was carried out by variation of the downcomer flow cross section. The results clearly show that the stability behaviour of the thermal hydraulic single heated channel model is very sensitive to the downcomer flow cross section. Consequently, BWR stability analysis should be performed always including the recirculation loop. Further, it can be concluded from the sensitivity investigations that the downcomer friction can be neglected in further investigations.

Furthermore, the numerical integration method was presented for the thermal hydraulic single heated channel model in a close neighborhood of two points on the SB. The dynamical behavior predicted by the semi-analytical bifurcation analysis could be confirmed independently by the numerical integration.

\section{NOMENCLATURE}

$\rho_{g}^{*}$ vapor density, $\rho_{f}^{*}$ liquid density, $g^{*}$ gravitational constant, $\mathrm{v}_{0}^{*}$ reference velocity, $\Delta h_{f g}^{*}$ vapor-liquid enthalpy difference, $\Delta \rho^{*}$ liquid-vapor density difference, $h_{\text {sat }}^{*}$ liquid saturation enthalpy

Heated channel: $h_{\text {inlet }}^{*}$ inlet enthalpy, $v_{\text {inlet }}^{*}$ inlet velocity, $A_{\text {inlet }}^{*}$ cross section, $L^{*}$ length, $D^{*}$ hydraulic diameter, $f_{1 \Phi}^{*}$ single phase friction factor, $h_{\infty, 1 \Phi}^{*}$ clad surface heat transfer coefficient of the single phase region, $\xi_{h}^{*}$ heat perimeter of the clad, $q_{1 \Phi}^{n * *}$ single phase wall heat flux,

Downcomer: $\Delta P_{\text {extern }}$ external pressure drop, $\dot{m}_{\text {tot }}(t)$ total mass flow, $h_{\text {doc inlet }}^{*}$ downcomer inlet enthalpy, $A_{d o c}^{*}$ downcomer cross section, $D_{d o c}^{*}$ downcomer hydraulic diameter,

Dimensionless numbers: $N_{f 1 \Phi}$ single phase friction number, $\mathrm{Fr}$ Froude number, $N_{p c h}$ phase change number, $N_{\text {sub }}$ subcooling number

$$
\begin{aligned}
& N_{\text {sub }}=\frac{\left(h_{\text {sat }}^{*}-h_{\text {inlet }}^{*}\right)}{\Delta h_{f g}^{*}} \cdot \frac{\Delta \rho^{*}}{\rho_{g}^{*}}, \quad N_{p c h}=\frac{q^{*} \xi_{h}^{*} L^{*} \Delta \rho^{*}}{A_{\text {inlet }}^{*} \Delta h_{f g}^{*} \rho_{g}^{*} \mathrm{v}_{0}^{*}}, \\
& A_{o l}=\frac{A_{\text {inlet }}^{*}}{A_{d o c}^{*}}, \quad D_{o l}=\frac{D^{*}}{D_{d o c}^{*}}, \quad N_{f 1 \Phi}=\frac{f_{1 \Phi}^{*} L^{*}}{2 D^{*}}, \quad F r=\frac{\mathrm{v}_{0}^{* 2}}{g^{*} L^{*}}
\end{aligned}
$$

\section{ACKNOWLEDGEMENT}

The authors would like to express their appreciation to M. A. Zimmermann, the STARS project manager at the Laboratory for Reactor Physics and Systems Behaviour, Paul Scherrer Institut, Switzerland for the warm hospitality received by the first author during his stay at PSI.

The authors would like to thank A.Dokhane (PSI) for his support and discussions. 
This work is sponsored by the Bundesministerium für Wirtschaft und Technologie (BMWi 1501290)

\section{REFERENCES}

[1] D. Hennig, "Boiling Water Reactor Stability Analysis-A Challenge for the Study of Complex Nonlinear Dynamical Systems,” PSI Annual Report 1996, Annex IV, Nuclear Energy and Safety.

[2] A. Dokhane, D. Hennig, R. Chawla, and Rizwan-uddin, "Nonlinear stability analysis of boiling water reactor on the basis of system codes and reduced order models," Proceedings of Annual Meeting on Nuclear Technology, Vol. 126, pp.15-19, 2003.

[3] A. Dokhane, "BWR Stability and Bifurcation Analysis using a Novel Reduced Order Model and the System Code RAMONA," Doctoral Thesis, EPFL, Switzerland, 2004.

[4] A. Dokhane, D. Hennig, Rizwan-uddin, and R. Chawla, "Semi-analytical Bifurcation Analysis of Two-phase Flow in a Heated Channel," International Journal of Bifurcation and Chaos, 15(8), 2005.

[5] A. Dokhane, D. Hennig, R. Chawla, and Rizwan-uddin, "Nuclear-Coupled ThermalHydraulic Nonlinear Stability Analysis Using a Novel BWR Reduced Order Model: part 1- The Effects of Using Drift Flux Versus Homogeneous Equilibrium Models," Proc. of $11^{\text {th }}$ International Conference on Nuclear Engineering, Tokyo, Japan, 2003.

[6] A. Dokhane, D. Hennig, R. Chawla, and Rizwan-uddin, "Nuclear-Coupled ThermalHydraulic Nonlinear Stability Analysis Using a Novel BWR Reduced Order Model: part 2stability limits of in-phase and out-of-phase modes; Proc. of $11^{\text {th }}$ International Conference on Nuclear Engineering,” Tokyo, Japan, 2003.

[7] M. Miro, D. Ginestar, D. Hennig, and G. Verdu, "On the Regional Oscillation Phenomenon in BWR's," Progress in Nuclear Energy, Vol. 36; No. 2; pp. 189-229, 2000.

[8] M. Tsuji, K. Nishio, M. Narita, Y. Ogawa, and M. Mori, "Stability Analysis of BWR's Using Bifurcation Theory," J. Nucl. Sciences and Technology, 30, (11), pp. 1107-1119, 1993.

[9] J. Munoz-Cobo, M. Podowski, and S. Chiva, "Parallel channel instabilities in boiling water reactor systems: boundary conditions for out of phase oscillations; annals of nuclear energy," 29, 1891-1917, 2002.
[10] Rizzwan-uddin, "Turning points and sub- and supercritical bifurcations in a simple BWR model," to appear in Nuclear Engineering and Design; 2005.

[11] J. Guggenheimer and P. Holmes, “Nonlinear Oscillation, Dynamical Systems, and Bifurcation in Vector Fields," Applied Mathematical Sciences 42, Springer Verlag, 1984.

[12] A. Neyfeh and B. Balachandran, "Applied nonlinear dynamics: analytical, computational, and experimental methods,” John Wiley \& Sons, Inc., New York, 1995.

[13] D. Hassard, D. Kazarinoff, and Y-H Wan, "Theory and Applications of Hopf Bifurcation," Cambridge University Press, New York, 1981.

[14] K. Hashimoto, "Linear Modal Analysis of Outof-Phase Instability in Boiling Water Reactor Cores,” Ann. Nucl. Energy, 20, 12, 789-797, 1993.

[15] K. Hashimoto, A. Hotta, and T. Takeda, "Neutronic Model for Modal Multichannel Analysis of Out-of-Phase Instability in Boiling Water Reactor Cores,” Ann. Nucl. Energy, 24, 2, 99-111, 1997.

[16] T. Lahey, Jr. and J. Moody, "The ThermalHydraulics of a Boiling Water Nuclear Reactor," Second Edition; American Nuclear Society, La Grange Park, Illinois USA, 1993.

[17] A. Karve, "Nuclear-Coupled Thermal-hydraulic Stability Analysis of Boiling Water Reactors," Ph.D. Dissertation, Virginia University, USA, 1998.

[18] V. Garcia i Llorens, C. Lange, D. Hennig, R. Mirò, and G. Verdu, "Feedback Reactivity Calculations Using Exact Expression from LAMBDA-REAC code," Proc. of PHYSOR2006, Vancouver, BC, Canada, 2006. 